\title{
A PROBABLE ASSOCIATION OF ACUTE DYSTONIA WITH GEMIFLOXACIN ADMINISTRATION
}

\author{
DINESH DUTT SHARMA, ASHISH AGGARWAL, RAVI C. SHARMA, RAMESH KUMAR
}

\section{ABSTRACT}

Gemifloxacin is a recently introduced fluoroquinolone antibiotic frequently used for its broad spectrum and once-daily dosing. Fluoroquinolones are associated with various neuropsychiatric side effects, such as seizures, insomnia, confusion, lightheadedness, psychosis, paranoia and hallucinations. We report a case of a 36-year-old woman given gemifloxacin for an upper respiratory tract infection who developed acute dystonia on the third day following therapy initiation. The clinical implications are discussed.

Key words: Acute dystonia, fluoroquinolones, gemifloxacin, neuropsychiatric effects DOI: $10.4103 / 0019-5359.59989$

\section{INTRODUCTION}

Gemifloxacin is a broad-spectrum fluoroquinolone antibiotic recently introduced into the Indian market. Besides common side effects like rash, diarrhea, nausea, vomiting, it has been reported to be associated with neurotoxicity syndromes such as encephalopathy and seizures. ${ }^{[1,2]}$ Other central nervous system (CNS) side effects like tremors, restlessness, anxiety, lightheadedness, confusion, hallucinations, paranoia, depression, insomnia and, rarely, suicidal thoughts have been reported with other fluoroquinolones. ${ }^{[2]}$

Department of Psychiatry, Indira Gandhi Medical

College, Shimla, Himachal Pradesh, India

Correspondence:

Dr. Dinesh Dutt Sharma

Department of Psychiatry,

IGMC, Shimla, India

E-mail: dineshdutt@yahoo.com
These have, however, not been reported with gemifloxacin. The mechanism of action for this class involves inhibition of gamma-amino butyric acid (GABA) binding to receptors in the brain. ${ }^{[3]}$ Other neurotransmitters like N-methylD-aspartic acid (NMDA) and adenosine have also been implicated in the pathogenesis of CNS side effects.

We report a case of probable acute dystonia following initiation of gemifloxacin.

\section{CASE REPORT}

Prior to presentation, a 36-year-old woman was treated for an upper respiratory tract infection with loratidine. Three days later, gemifloxacin $320 \mathrm{mg} /$ day was added. After the first dose of gemifloxacin, the patient reported difficulty in sleeping. On the third day of therapy, the patient developed involuntary 
twisting movements of the upper extremities, progressing to the lower extremities within 30 minutes. Restlessness was associated. The patient was brought in the casualty and a diagnosis of acute dystonia, drug induced, was made. Her past medical and family history for any movement disorder or any other neurological or psychiatric illnesses was not significant. There was no history of any head injury; or any illicit drug intake; or taking any other medication, including cough syrups; or any other treatment.

Neurological examination revealed a fully conscious and alert patient in moderate distress. Her vitals were stable, though her heart rate was increased to 100 beats per minute. Deep tendon reflexes were brisk, direct and indirect light reflexes were reactive and pupils were isocoric. Sustained muscle contractions in the hands, forearm, arm; and also in the lower limbs, involving muscles of the foot, calf and the thigh, were observed. All the 4 extremities were in the extensor tonus. The dystonic movements were generalized, involving all the 4 limbs. The axial musculature was spared from the dystonic movements. No other abnormal involuntary movements were observed. The patient did not report any inner motor restlessness (suggestive of akathisia), and her restlessness was secondary to anxiety because of abnormal posture. Findings from fundus examination were within normal limits (WNL). Results of diagnostic workup, including complete blood counts, electrolytes, liver and renal function tests, urinalysis, chest x-ray, computed tomography (CT) scan of the head (plain and contrast) and electroencephalogram (EEG), were all WNL. Electrocardiogram done at that time revealed sinus tachycardia.
The patient was given promethazine $50 \mathrm{mg}$ intravenously, and the dystonia resolved within 5 to 10 minutes. A diagnosis of gemifloxacininduced dystonia was made. The Naranjo adverse drug probability score was ' 6 ,' correlating with a probable relationship. ${ }^{[4]}$ The patient has been maintaining well since then without any recurrence of dystonia for the past 8 months of follow-up.

\section{DISCUSSION}

A case for gemifloxacin-induced acute dystonic reaction was consistent with patient presentation and temporal connection with pharmacotherapy initiation. The patient was conscious, had no prior medical or psychiatric problems, had no symptom progression and remitted with promethazine. Promethazine was used for the management of dystonia as antihistaminics and anticholinergics are considered drugs of choice for the management of dystonia, though there are occasional case reports of them being causing dystonia. ${ }^{[5,6]}$

Administration of loratidine as a factor in the causation of dystonia was ruled out; no problems were reported with its present or past usage prior to the addition of gemifloxacin to the regimen. Moreover, loratidine was restarted without any untoward effects. Prior to this course of therapy, the patient was fluoroquinone naïve. There was no other associated history to suggest any other cause for dystonia. Rechallenge with gemifloxacin was not attempted; therefore the diagnosis was presumptive.

Most of the neuropsychiatric side effects have been reported to occur between days 2 and 8 
after starting fluoroquinolones, which is well within the time frame of our case, in which dystonia occurred on the $3^{\text {rd }}$ day of start of gemifloxacin. ${ }^{[7]}$ Besides this, drug-induced dystonia has been reported to occur generally within 7 days of starting any drug or increasing the dose of any drug. ${ }^{[8]}$

Dystonia is characterized by sustained contraction/ spasm of a group of muscles. It can occur secondary to a variety of drugs, especially antipsychotic medications, antiemetic drugs and some antidepressants too. Other drugs that have been reported include buspirone, diazepam, sumatriptan, phenylpropanolamine and ecstasy. ${ }^{[8]} A$ review of the literature revealed that previous reports of quinolone-mediated movement disorders were scarce and included a report of orofacial dyskinesia and chorea associated with ciprofloxacin use..$^{[9,10]}$

The pathogenesis of acute dystonia is still unclear. Since dystonia is most commonly associated with antipsychotic medications, it has been suggested that blockage of Dopamine D2 receptors in the caudate, putamen and globus pallidus is partly responsible for causing acute dystonia. ${ }^{[11]}$

Besides this, impairments in CNS GABA levels have been reported to be involved. ${ }^{[12]}$

The structural component of the fluoroquinolone molecule, believed to be responsible for improved gram-positive activity, is also believed to be implicated in the production of CNS adverse effects. Inhibition of brain GABA receptor binding appears to be a strong indicator of CNS activity, though NMDA receptor binding has also been implicated. ${ }^{[13]}$ Antagonistic effects at the benzodiazepineGABA receptor have been demonstrated. ${ }^{[14]}$

These could be the mechanisms involved in the pathogenesis of dystonia caused by fluoroquinolones.

Patients with acute dystonic reactions have been reported misdiagnosed with a variety of conditions, ranging from encephalitis to seizures and tetanus. ${ }^{[15,16]}$ Misdiagnosis results in delayed treatment, increased treatment costs and unnecessary medication exposure.

This case highlights the need for clinicians to be cautious when evaluating patients with dystonias and take detailed treatment histories to avoid unnecessary delays in treatment, as well as increased treatment costs and medication exposure. Continued vigilance is recommended as increasing use of gemifloxacin and other fluoroquinolones for a variety of infectious diseases may result in increased likelihood of adverse effects, which are infrequently reported.

\section{REFERENCES}

1. Barrett MJ, Login IS. Gemifloxacin-associated neurotoxicity presenting as encephalopathy. Ann Pharmacother 2009;43:782-4.

2. Factive (Gemifloxacin mesylate) prescribing information. Oscient Pharmaceuticals Corporation. Available from: http://www.factive. com/pdf/prescribing_info.pdf. [cited in 2008].

3. Tsuji A, Sato H, Kume Y, Tamai I, Okezaki E, Nagata $\mathrm{O}$, et al. Inhibitory effects of quinolone antibacterial agents on gamma-aminobutyric acid binding to receptor sites in rat brain membranes. Antimicrob Agents Chemother 1988;32:190-4. 
4. Naranjo CA, Busto $U$, Sellers EM, Sandor $P$, Ruiz I, Roberts EA, et al. A method for estimating the probability of adverse drug reactions. Clin Pharmacol Ther 1981;30:239-45.

5. DeGrandi T, Simon JE. Promethazine-induced dystonic reaction. Pediatr Emerg Care 1987;3:912.

6. Casey DE. Neurolepticinduced acute dystonia. In: Lang AE, Weiner WJ, editors. Druginduced movement disorders. Mount Kisco, NY: Futura; 1992. p. 2140.

7. James EA, Demian AZ. Acute psychosis in a trauma patient due to ciprofloxacin. Postgrad Med J 1998;74:189-90.

8. Van Harten PN, Hoek HW, Kahn RS. Acute dystonia induced by drug treatment. BMJ 1999;319:623-6.

9. Lee $\mathrm{CH}$, Cheung RT, Chan TM. Ciprofloxacin induced oral facial dyskinesia in a patient with normal liver and renal function. Hosp Med 2000;61:142-3.

10. Azar S, Ramjiani A, Van Gerpen JA. Ciprofloxacin induced chorea. Mov Disord 2005;20:513-4.
11. Rupniak NM, Jenner P, Marsden CD. Acute dystonia induced by neuroleptic drugs. Psychopharmacology 1986;88:40319.

12. Levy LM, Hallett M. Impaired brain GABA in focal dystonia. Ann Neurol 2002;51:93-101.

13. Lode $\mathrm{H}$. Potential interactions of the extended spectrum fluoroquinolones with the CNS. Drug Saf 1999;21:123-35

14. Unseld E, Ziegler G, Gemeinhardt A, Janssen U, Klotz U. Possible interaction of fluoroquinolones with the benzodiazepine GABA receptor complex. Br J Clin Pharmacol 1990;30:63-70.

15. Cezard C, Nisse P, Quaranta S, Peucelle $D$, Mathieu-Nolf M. Acute dystonia from metoclopramide in children. Therapie 2003;58:367-70.

16. Yis $U$, Ozdemir D, Duman M, Unal N. Metoclopramide induced dystonia in children: Two case reports. Eur J Emerg Med 2005;12:117-9.

Source of Support: Nil. Conflict of Interest: None declared.

\section{Author Help: Reference checking facility}

The manuscript system (www.journalonweb.com) allows the authors to check and verify the accuracy and style of references. The tool checks the references with PubMed as per a predefined style. Authors are encouraged to use this facility, before submitting articles to the journal.

- $\quad$ The style as well as bibliographic elements should be $100 \%$ accurate, to help get the references verified from the system. Even a single spelling error or addition of issue number/month of publication will lead to an error when verifying the reference.

- $\quad$ Example of a correct style Sheahan P, O'leary G, Lee G, Fitzgibbon J. Cystic cervical metastases: Incidence and diagnosis using fine needle aspiration biopsy. Otolaryngol Head Neck Surg 2002;127:294-8.

- Only the references from journals indexed in PubMed will be checked.

- $\quad$ Enter each reference in new line, without a serial number.

- Add up to a maximum of 15 references at a time.

- If the reference is correct for its bibliographic elements and punctuations, it will be shown as CORRECT and a link to the correct article in PubMed will be given.

- If any of the bibliographic elements are missing, incorrect or extra (such as issue number), it will be shown as INCORRECT and link to possible articles in PubMed will be given. 